\title{
EINIGE GEDANKEN ZUR TONARTENORDNUNG IN SCHUBERTS LIEDERZYKLEN
}

\author{
Kurt von Fis cher (Zürich)
}

Als Zyklen im engeren Sinne des Wortes sind, auch von den Textvorlagen her gesehen, nur Die schöne Müllerin und Winterreise zu verstehen, während Schwanengesang, mit Ausnahme des bekanntlich erst nachträglich vom Verleger zugefügten letzten Liedes, Die Taubenpost, eine offene Reihung von sieben Rellstab- und sechs Heinetexten darstellt. Trotzdem scheint es, als ob Schubert zumindest die HeineLieder als Zyklus verstanden haben dürfte. Dies geht daraus hervor, dass der Komponist am 2. Oktober 1828 die Heinevertonungen dem Leipziger Verleger Probst, zusammen mit dem Streichquintett D 956 und mit den drei Klaviersonaten D 958-960 separat angeboten hat. ${ }^{1}$ Sie seien deshalb, nicht zuletzt auch ihrer in jüngster Zeit nicht ganz unbestrittenen Reihenfolge wegen, in unsere Betrachtungen mit einbezogen. Die Frage, die in den folgenden als Essay zu verstehenden Untersuchungen zu stellen ist, lautet: Gibt es bei Schubert eine den Textinhalten entsprechende Zyklik der Tonarten und wie ist diese allenfalls beschaffen. Hierbei sind die motivischen Bezüge, die zuweilen zwischen einzelnen Liedern $\mathrm{zu}$ beobachten sind, nur teilweise mitberücksichtigt.

Vorbemerkung zur Technik der Tonartenverknüpfung: In Schuberts Harmonik spielen bekanntlich nicht nur Tonika-Dominant- und Subdominantverhältnisse, sondern vor allem auch Terzverwandtschaften und damit das Prinzip gemeinsamer Töne als Verbindungsmöglichkeiten zweier Tonarten eine wichtige Rolle. Im Zusammenhang mit den vorliegenden Untersuchungen sei daher postuliert, dass für Schubert z. B. die Verbindung eines Liedes in G-dur mit einem folgenden in a-moll, trotz einfacher Umdeutungsmöglichkeit von a-moll als zweite Stufe von G-dur, einen geringeren Vermittlungsgrad bedeuten kann als z. B. der Wechsel von A-dur zu C-dur

1 Vgl. hierzu Otto Erich Deutsch, Franz Schubert, Thematisches Verzeichnis seiner Werke in chronologischer Folge, Neuausgabe in deutscher Sprache bearbeitet und herausgegeben von der Editionsleitung der Neuen SchubertAusgabe und Werner Aderhold, Bärenreiter 1978. 
(mit gemeinsamen Ton e). Hierbei vermögen funktionsmässig entferntere Beziehungen mittels gemeinsamer Töne bedeutungsvolle Farb -und Charakterwechsel zu bewirken.

\section{Die schöne Müllerin}

Wenn auch Schubert nicht alle Gedichte von Wilhelm Müllers Zyklus vertont hat, so ergibt sich doch die Zyklik dieser Lieder von der Textvorlage her im Sinne einer durchgehenden Handlung vom ersten bis zum zwanzigsten Lied. ${ }^{2}$ Dem scheint zunächst nur teilweise eine auch in der Tonartenfolge nachweisbare Gliederung und Kontinuität $\mathrm{zu}$ entsprechen. Mit Bezug auf die Textinhalte der einzelnen Lieder und Liedgruppen jedoch lassen sich, wie im folgenden zu zeigen versucht wird, gewisse Prinzipien erkennen, welche die Tonartswahl und -folge die Lieder als keinswegs beliebig erscheinen lassen.

Zunächst seien die Tonarten der Lieder in einer Tabelle aufgelistet, wobei hier bloss die Anfangs- und Schlussklänge berücksichtigt sind. Diese sind denn auch für den tonartlichen Stellenwert eines Liedes innerhalb des Zyklus von besonderer Bedeutung: ${ }^{3}$

$\begin{array}{rccccccccccc}\text { Nr. } 1 & 2 & 3 & 4 & 5 & 6 & 7 & 8 & 9 & 10 & 11 & 12 \\ \text { B } & \text { G } & \text { C } & \text { G } & \text { a } & \text { H } & \text { A } & \text { C } & \text { A } & \text { A-a } & \text { D } & \text { B } \\ 13 & 14 & 15 & 16 & 17 & 18 & 19 & 20 & & & & \\ \text { B } & \text { c } & \text { g-G } & \text { h } & \text { H-h } & \text { e } & \text { g-G } & \text { E } & & & & \end{array}$

Tonartlicher Ausgangspunkt des Zyklus ist B-dur (Nr. 1: Das Wandern), eine Tonart, die von Schubert zunächst nicht weiter verfolgt wird. Als Bindeglied zum folgenden G-dur (Nr. 2: Wohin?) kann einzig der den beiden Grundakkorden gemeinsame Ton d verstanden werden. Damit erweist sich das erste Lied auch tonartlich als ein dem Zyklus vorangestelltes Motto, das die Wanderthematik exponiert. Die Lieder Nr. 2, 3 und 4 gehören vom Text her zusammen, zentrale Begriffe sind Bächlein und Wasser. Tonartlich spiegelt sich dies in der engen Beziehung G-C-G, wobei die Frage Wohin (Nr. 2) dominantisch auf das folgende Halt! (Nr. 3) zielt. Nr. 5 (Am Feierabend) steht, bezogen auf das vorherige G-dur von Nr. 4 (Danksagung an den Bach), isoliert in a-moll: Die Reise ist unterbrochen. Die Wahl von a-moll als Paralleltonart des C-dur Nr. 3 ergibt sich daraus, dass ja auch dieses Lied ein Halt! in der Wanderung beinhaltet. Nr. 6 in H-dur (Der Neugierige) ist tonartlich sowohl vom vorherigen wie auch vom folgenden

2 Der vollständiger Gedichtzyklus ist leicht greifbar bei A. Feil, Franz Schubert, Die schöne Müllerin - Winterreise, Stuttgart 1975, S. 151-162.

${ }^{3}$ Die im Deutsch-Verzeichnis auf S. 489 genannten Transpositionen der Lieder Nr. 16 und 18-20 sind hier nicht berücksichtigt, da diese vermutlich nicht auf Schubert, sondern auf den Sänger J. M. Vogl zurückgehen. 
Lied (A-dur) abgehoben. Es ist dies das erste Stück des Zyklus in hohen Kreuztonarten, die erst wieder in den Nummern 17 (H-dur) und 20 (E-dur) und zudem im zweiten Teil von Nr. 18 (E-dur) erscheint. Könnte dieses $\mathrm{H}$-dur als erster Hinweis auf diese letzten Lieder verstanden werden? Das Bächlein meiner Liebe ist »heut so stumm" und »wunderlich «!

Die Nummern 7 bis 9 und teilweise auch Nr. 10 sind reine Strophenlieder, eigentliche Lieder des wandernden Gesellen:4 Ungeduld, Morgengruss, Des Müllers Blumen und Tränenregen. Mit dem dreimaligen A-dur (Nr. 7, 9 und 10) weist Schubert auf das Gemeinsame dieser Stücke. Dass Nr. 8 (Morgengruss) nicht auch in A, sondern, mit gemeinsamem Ton e, in C steht, lässt sich einerseits mit dem Varietas-Prinzip, andererseits aber mit der diesem Lied eigenen direkten Rede (»Guten Morgen, schöne Müllerin«) begründen. Auch Nr. 10 (Tränenregen) beginnt als reines Strophenlied und, wie Nrn. 7 und 9, in A-dur. Mit der letzten Strophe (»Da gingen die Augen mir über«) verdunkelt sich der heitere Liedton zu moll: subjektive Betroffenheit und düstere Vorahnung des Kommenden, zugleich aber auch Hiatus vor dem folgenden Lied. Die inhaltliche Beziehung von Nr. 11 (Mein!) zu Nr. 1 ist nicht nur in der rhythmischen Struktur evident, ${ }^{5}$ sie ist auch mit der Tonart B-dur des Mittelteils angedeutet. Hier könnte der Zyklus zu Ende sein: Die Wanderung schliesst mit der Liebeserfüllung. Doch nun folgt mit N. 12 (Pause) die Wende. Nochmals (wie schon in Nr. 1) setzt Schubert mit B-dur ein, jetzt aber nicht mehr im Wanderton, sondern mit kurzatmigen Rhythmen und mit Eindunkelung zu es-moll (»Warum liess ich das Band auch hängen so lang? «).

In den Texten der folgenden fünf Lieder (Nrn. 13-17), anknüpfend an die zweite Zeile von $\mathrm{Nr} .12$ (»Hab sie umschlungen mit einem grünen Band«) erscheint die grüne Farbe als Leitgedanke: grün wohl nur mehr ironisch als Farbe der Hoffnung verstanden. Schubert fasst tonartlich die Nrn. 13-15 (B-Tonarten) und 16-17 (Kreuztonarten) zusammen: B-c-g und h-H (h). Damit führt er zunächst, als Ausdruck der beiden Liedern gemeinsamen Lautenthematik, das B-dur von $\mathrm{Nr}$. 12 weiter, setzt jedoch in Nr. 14 (Der Jäger) das c-moll vom vorangehenden B-dur ab: Signal für das drohende Unheil. Im letzten Teil von Nr. 15 (Eifersucht und Stolz) verwandelt sich g-moll zu G-dur. Damit ist eine enge terzverwandtschaftliche Verbindung (zwei gemeinsame Töne!) zum h-moll von Nr. 16 (Die liebe Farbe) gegeben. Im Nachhinein erscheint damit der G-dur Schluss von $\mathrm{Nr}$. $15 \mathrm{zu}$ brüchiger Heiterkeit ironisiert (»Er schnitzt bei mir sich eine Pfeif aus Rohr und bläst den Kindern schöne Tänz und Lieder, vor «).

In Nr. 16 ist zum erstenmal das Ziel der Wanderung eindeutig angesprochen: Das Grab (3. Strophe: „Grabt mir ein Grab im Wasen«). Die

4 Vgl. Feil, S. 75.

5 Ibid., S. 79. 
Tonart h-moll verbindet sich hier mit Ausweglosigkeit, signalisiert mittels durchgehender Tonrepetition fis. Das H-dur von $\mathrm{Nr} .17$ ist mit seinen moll-Eindunkelungen als ironische Umkehrung von $\mathrm{Nr} .16 \mathrm{zu}$ deuten: Die liebe Farbe in moll, Die böse Farbe in Dur. Das h dieser beiden Stücke führt als Dominante unmittelbar zum folgenden Lied Nr. 18 (Trockene Blumen) in e-moll: Die grüne Farbe ist nun zum Grabeshügel geworden, der scheinbare Aufbruch von Nr. 17 (»Ich möchte ziehen in die Welt hinaus «) entpuppt sich als blosser Schein. Mit e ist nun zugleich der Gegenpol (Tritonus) zur Ausgangstonart sowohl des ersten als auch des zweiten Teils (Nrn. 1 und 12) des Zyklus erreicht. Die im zweiten Teil von Nr. 18 erscheinende Tonart E-dur weist auch schon auf das letzte Lied (Baches Wiegenlied). Bevor aber das Ende erreicht ist, erinnert Nr. 19 (Der Müller und der Bach) nochmals an Vergangenes, die g-G-Tonart zugleich an den wilden Bach von Nr. 15 (g-G) und an das Bächlein der Lieder Nr. 2 und 4 (G).

Tonartlich verlaufen die beiden Teile des Zyklus - durchaus den Textinhalten entsprechend - gegenläufig: in den Liedern $\mathrm{Nr}$. 1-11 von B-dur über G, C, a (H), A nach D (mit Mittelteil in B), in den Nrn. $12-20$ von $B-d u r$ über $b$-Tonarten und in Kreuztonarten umschlagend zum E-dur Schlusslied, das metrisch und motivisch Ruhe des Todes und Auflösung ins All beinhaltet. ${ }^{6}$

Die Tonartsfolge der einzelnen Lieder der Schönen Müllerin ist nur teilweise eine im üblichen Sinne funktional logische, sie beruht vielmehr auf den durch die Textinterpretation gegebenen Assoziationen, die von Schubert nicht abbildhaft tonmalerisch, sondern im Sinne eigenständiger musikalischer Strukturen realisiert sind.

\section{Winterreise}

Im Gegensatz zur Schönen Müllerin beinhalten die Texte der Winterreise kein eigentliches Geschehen, sondern vielmehr eine Reihe von Stationen der Ausweglosigkeit und Verzweiflung. Mehr als die Hälfte der Liedtexte enthält die Worte Schnee und Eis, acht Lieder sprechen von Grab und Tod. So herrschen denn hier auch die mollTonarten vor. Wo dur erscheint, beinhaltet dieses nicht Helle, sondern zwielichtige Vision einer Traumwelt. Kann es in der Winterreise eine tonartlich zu begründende Zyklik geben? Diese Frage ist überdies deshalb zu stellen, weil Schubert in fünf Liedern (Nr. 6, 10, 12, 22, 24) die ursprüngliche Tonart nachträglich veränderte. Zudem hat der Komponist zunächst nur die 1823 von Wilhelm Müller veröffentlichten zwölf ersten Lieder vertont und erst einige Monate später, aus der in der zweiten Ausgabe von 1824 nun 24 Lieder umfassenden Sammlung,

6 Man vergleiche hierzu die eindrucksvolle Interpretation dieses Liedes bei Th. Georgiades, Schubert, Musik und Lyrik, Göttingen 1967, S. 305 ff. 
die im ersten Teil noch fehlenden Texte zu einem zweiten Teil zusammengefasst. Trotzdem sei der Versuch gewagt, zumindest einzelne zyklische Elemente in der Tonartenfolge des vollständigen Schubertschen Zyklus aufzuspüren. ${ }^{7}$ Hier zunächst die Liste der Tonarten in der endgültigen Fassung (auf die Frage der Transpositionen wird weiter unten noch einzugehen sein):

$\begin{array}{cccccccccccc}1 & 2 & 3 & 4 & 5 & 6 & 7 & 8 & 9 & 10 & 11 & 12 \\ \text { d } & \text { a } & \text { f } & \text { c } & \text { E } & \text { e } & \text { e } & \text { g-G } & \text { h } & \text { c } & \text { A-a } & \text { h } \\ 13 & 14 & 15 & 16 & 17 & 18 & 19 & 20 & 21 & 22 & 23 & 24 \\ \text { Es } & \text { c } & \text { c } & \text { Es } & \text { D } & \text { d } & \text { A } & \text { g } & \text { F } & \text { g } & \text { A } & \text { a }\end{array}$

Die Lieder Nr. 1 und 2 (Gute Nacht, Die Wetterfahne) sowie Nr. 3 und 4 (Gefrorene Tränen, Erstarrung) gehören textlich zusammen: Abschied, Sturm, dann Eis und Schnee. Schubert vertont diese vier Lieder einerseits als je zwei moll-dominantisch aufeinander bezogene Paare (d-a und f-c), andererseits sind alle vier Stücke durch gemeinsame Töne der jeweiligen Anfangs- und Schlussakkorde miteinander verbunden (Töne a und c). Nun folgt, vom vorangehenden Lied tonartlich geschieden, eine Gruppe von drei Stücken in e (Der Lindenbaum, Wasserflut, Auf dem Flusse): Die zwei Facetten von Traum und Realität stehen mit dur- und moll-Färbung auf demselben Grundton. Auch motivisch eng verbunden sind die Lieder Nr. 5 und 6, während in den Nrn. 6 und 7 der Begriff Wasser thematisiert ist. Rückblick (Nr. 8) erscheint in g-moll; es ist dies ein Lied, das sowohl vom Text als auch von der Tonart her auf die Nrn. 1 und 4 zurückblickt (g als moll-Subdominante von $d$ und als moll-Dominante von c). Es folgt Nr. 9 (Irrlicht) in h-moll. Wenn auch mit dem vorangehenden g-moll durch den Ton $d$ verbunden, ist es doch mit seinem von einer b- zur Kreuztonart hinüberwechselnden Charakter als ein das ironisch zum Ziel führende Irren beinhaltender Einschub $\mathrm{zu}$ verstehen. Man ist zugleich versucht, die Tonart h-moll mit der Grabesthematik von Nr. 16 (Die liebe Farbe) aus Die schöne Müllerin in Verbindung zu bringen. Erscheint doch auch hier in der Winterreise das Wort Grab zum erstenmal im Text (»Jeder Strom wird's Meer gewinnen, jedes Leiden auch sein Grab.«). Nr. 10 (Rast) greift, tonartlich ohne Verbindung mit dem vorangegangenen Lied, mit c-moll auf Nr. 4 zurück: zwei rhythmisch kontrastierende Aspekte

7 Der Vorschlag Ch. Kellers, die Lieder in der Reihenfolge der Textausgabe von. 1824 vorzutragen ist zwar interessant, doch würde damit die von Schubert komponierte Liedfolge und damit die intendierte Zyklik zerstört. Vgl. hierzu Ch. Keller, »Musik als schlechtes Theater? Gedanken zu einer nicht-einfühlenden Darstellungsweise von Musik - angeregt durch Brechts Schauspielkritik«, in Aspekte der musikalischen Interpretation - Sava Savoff zum 70. Geburtstag, hersg. von H. Danuser und Ch. Keller, Hamburg 1980, S. $91 \mathrm{ff}$. 
verzweifelter Resignation. Auch zum Beginn des folgenden Liedes (Frühlingstraum) in A-dur (mit moll-Einfärbungen) fehlt eine direkte Verbindung. Ähnlich wie schon in Der Lindenbaum stehen einander in Nr. 11 Traumesvisionen (Dur) und Realitäten (moll) gegenüber. Den Abschluss des ersten Teiles der Winterreise bildet Einsamkeit (Nr. 12) in h-moll, d. h. wiederum ohne direkten Anschluss an die a-Tonart von Nr. 11. Zugleich weist dieses Lied tonartlich auf Nr. 9 (Irrlicht) zurück: Einsamkeit ist identisch mit der Verführung durch das Irrlicht.

Im zweiten Teil des Zyklus werden, wie schon zu Beginn des ersten, vier Lieder (Nr. 13-16), diesmal noch enger, tonartlich zusammengeschlossen: Die Post (Es-dur mit es-moll Teilen), die zwei in c-moll stehenden, auch vom Text her eng zusammengehörigen Stücke Der greise Kopf (Nr. 14) und Die Krähe (Nr. 15) sowie Letzte Hoffnung (Nr. 16), das, nun allerdings zu Beginn mit stark verfremdetem Es-dur die Tonart von Nr. 13 aufgreift: Jede in Die Post noch mögliche Hoffnung ist zunichte geworden. Tonartlich deutlich abgehoben von Nr. 16 sind die folgenden, in D-dur bezw. d-moll stehenden Lieder Im Dorfe (Nr. 17) und Der stürmische Morgen (Nr. 18), die vom Text her gesehen insofern zusammenhängen, als beide Stücke beschreibende Elemente und damit eine gewisse Distanz zum Subjekt aufweisen. Nr. 19 (Täuschung) steht in der Dominante a der vorangehenden zwei Lieder. Die Auftaktnote e hat a,ber gegenüber dem Schlusston d von Nr. 18 eine rückungsartige Wirkung im Sinne von Täuschung. Zugleich verbindet die Tonart A-dur dieses Lied mit Frühlingstraum (Nr. 11), dessen Inhalt ebenfalls als Irrealität und damit als Täuschung zu bezeichnen war. Nun folgt wiederum eine tonartlich zusammengehörige Gruppe, diesmal von drei Liedern in g-moll, F-dur und nochmals g-moll, die sowohl zum vorangehenden als auch zum folgenden Stück (Nr. 23) keine direkte Verbindung aufweisen. Innerhalb dieser Gruppe steht das mittlere Lied Das Wirtshaus (Nr. 21) insofern isoliert als der F-dur Akkord keine gemeinsamen Töne mit den umgebenden g-moll Grundakkorden von Nr. 20 (Der Wegweiser) und Nr. 22 (Mut) zeigt. Die Wahl von $\mathrm{F}$ findet vermutlich eine Erklärung durch den Hinweis von Georgiades auf den Zusammenhang der Liedmelodik mit dem Kyrie der Totenmesse, das ebenfalls im F-Modus steht. ${ }^{8}$ Auffallend ist die Wahl der gleichen Tonart für die das mittlere Lied Nr. 21 umschliessenden Nrn. 20 und 22.9 Beide Stücke sind überdies durch ähnliche Motivik (zwei auftaktig diatonisch ansteigende 16-tel) und durch den moll-dur-Wechsel miteinander verbunden. Eine Deutung bietet sich an, wenn man beide als resignierte (Nr. 20) bzw. ironische (Nr. 22) Facetten der gleichen Verzweiflung versteht. Eine Bestätigung dieser Absicht könnte allenfalls darin gesehen werden, dass Schubert

$\checkmark$ Vgl. Georgiades, S. 377.

9 Nr. 22 stand ursprünglich in a-moll, vgl. dazu die unten folgenden Ausführungen. 
nur gerade hier die Müllersche Reihenfolge durch Umstellung von Nebensonnen (Nr. 23) und Mut (Nr. 22) offenbar ganz bewusst abgeändert hat. Tonartlich getrennt von Nr. 22 steht Nr. 23 in A-dur. Damit ergibt sich eine Analogie zur tonartlich ebenfalls isolierten Lage von Lied Nr. 19, das, wie schon Nr. 11 (Frühlingstraum), ebenfalls in A stehend, Täuschung und Wahnvorstellungen beinhaltet.10 Schliesslich folg als Abschluss des Zyklus Der Leiermann in der Variantentonart von Nr. 23, in a-moll: nicht mehr Wahnvorstellung, sondern grause ausweglose Realität.

Wir fragen: Ist der Versuch, eine von den Texten her zu begründende tonartliche Ordnung der Winterreise im Sinne eines Zyklus nachzuweisen, geglückt? Wird die versuchte Deutung nicht dadurch fragwürdig, wenn man bedenkt, dass fünf Lieder ursprünglich in einer um einen, in Nr. 12 sogar um zwei Töne höher gelegenen Tonart gestanden haben? Wie sind diese Transpositionen zu deuten? Eine simple, wohl aber doch allzu simple Erklärung könnte sich aus der Stimmlage ergeben, indem in vier der fünf Lieder (Nrn. 6, 10, 12, 22) das hohe a durch die Transposition vermieden wird. Dem ist entgegenzuhalten, dass dieses a auch in Nr. 7 (Ungeduld) der Müllerinnenlieder und in Nr. 3 des Schwanengesangs (Frühlingssehnsucht) erscheint. Doch lassen sich auch von den Textinhalten und von der behaupteten zyklischen Ordnung der Lieder her Gründe für die Transpositionen anführen: Erst durch die Umsetzung von Nr. 6 (Wasserflut) von fis nach e ergibt sich die auch motivisch zu belegende enge Rückverbindung zu Nr. 5 (Der Lindenbaum). Ähnliches, wenn auch nicht motivisch begründbar, gilt für die von der Stimmlage her nicht zu begründende Transposition des Liedes Nr. 24 von $\mathrm{h}$ nach a, das damit in die Variante von Nr. $23 \mathrm{zu}$ stehen kommt. Überdies wird mit der Tonart a ein gleicher Abschluss der beiden Teile des mit einem Fragesatz (»Willst zu meinen Liedern deine Leier drehn?«) endenden und damit offenen Zyklus vermieden. Hier ist $\mathrm{zu}$ bedenken, dass Nr. 12 (Einsamkeit) in $d$ stand und erst nachträglich (in der Stichvorlage) nach $h$ transponiert wurde, was dann zur Tonartsänderung auch von $\mathrm{Nr} .24$ geführt haben dürfte. Mit dem h-moll von Nr. 12 wurde die inhaltlich $\mathrm{zu}$ begründende Trennung vom vorangehenden $\mathrm{A} / \mathrm{a}$ der $\mathrm{Nr}$. 11 vollzogen: Die Illusionen des Frühlingstraums sollen nicht dominantisch auf die resignierte Einsamkeit (Nr. 12) bezogen werden. - Nun bleiben noch die Transpositionen von Nr. 10 und $22 \mathrm{zu}$ erklären. Das ursprüngliche d-moll von $\mathrm{Nr}$. 10 hätte durch den gemeinsamen Ton d mit dem vorangehenden $\mathrm{h}$-moll von Irrlicht, aber durch den gemeinsamen Ton a mit dem A-dur von Nr. 11 eine offenbar nicht erwünschte Verbindung hergestellt, die der isolierten Stellung von Rast zwischen

10 Zur Deutung des Textes von Die Nebensonnen vgl. K. v. Fischer, "Zur semantischen Bedeutung von Textrepetitionen in Schuberts Liederzyklen«, in Schubert-Kongress Wien 1978, S. 340. 
Irrlicht und Frühlingstraum nicht entsprochen hätte. Dass Nr. 22 (Mut) von a nach g transponiert wurde, lässt sich schliesslich durch die oben erwähnte gemeinsame Motivik mit dem ebenfalls in g stehenden Wegweiser erklären. Zudem vermied Schubert damit die Tonart A-dur der dritten Strophe des Liedes, jenes A-dur, das den Nrn. 11, 19 und 23 ihren besonderen Charakter gibt. Die Tonartenfolge g-F-g/G-A-a entspricht wohl doch besser den Aussagen der Texte als die Folge g-F-a/A-A-h. Spekulationen? Vielleicht! Und doch scheinen mir gerade auch die Transpositionen auf Schuberts bewusstes Umgehen mit Tonarten $\mathrm{zu}$ weisen.

\section{Die Heine-Lieder}

In einem bemerkenswerten Aufsatz hat Harry Goldschmidt eine von Schubert ursprünglich anders konzipierte Abfolge der Heine.Lieder postuliert:11 Das Fischermädchen (As-dur) - Am Meer (C-dur) - Die Stadt (c-moll) - Der Doppelgänger (h-moll) - Ihr Bild (b-moll) - Der Atlas (g-moll). Die Logik von Goldschmidts Argumentation, im Hinblick auf die mit dieser Reihenfolge übereinstimmenden Ordnung der Gedichte bei Heine im Sinne einer Art von fortschreitendem Geschehen, scheint zwingend. ${ }^{12}$ Sie lässt sich nach Goldschmidt zudem mit einer dadurch entstehenden, gewissermassen logischen Tonartenfolge belegen: Aufsteigende Terz vom ersten zum zweiten Lied, Durmoll-Varianten im zweiten und dritten, absteigende Halbtonfolgen vom dritten bis fünften und absteigende Terz zwischen fünftem und letztem Lied. Gegen Goldschmidt spricht nun aber das vollständig erhaltene Autograph, in welchem die Lieder eindeutig in der bekannten Reihenfolge erscheinen. Gerade der Bruch mit der in Heines Texten vorgegebenen Ordnung entspricht dem musikalischen Denken Schuberts und findet eine Analogie in dem schon in der Winterreise beobachteten Wechsel von Realitätsvorstellungen, Traumes- und Wahnsinnsvisionen.

Der Zyklus beginnt mit einem wilden Ausbruch: Der Atlas. Es folgt, als starre Traumesvision gestaltet, Ihr Bild.

Das Fischermädchen beinhaltet Rückerinnerung und ist von Schubert mittels einer labilen Metrik als brüchig gewordenes barkarolenhaftes Lied gestaltet. ${ }^{13}$ In Die Stadt wird die ironisch heitere Stimmung des vorangegangenen Liedes zum »Nebelbild « getrübt (verminderte Septakkorde als Ostinato!). Am Meer knüpft, im Sinne einer nun ins Schmerzliche gewandten Erinnerung, an Das Fischermädchen an. Der Zyklus schliesst mit den Wahnvorstellungen des Doppelgängers.

11 H. Goldschmidt, "Welches war die ursprüngliche Reihenfolge in Schuberts Heine-Liedern? ", in Deutsches Jahrbuch der Musikwissenschaft für 1977, S. $52 \mathrm{ff}$.

12 H. Heine: Buch der Lieder - Die Heimkehr, Nrn. 8, 14, 16, 20, 23, 24.

13 Vgl. Georgiades, S. 77. 
Entspricht diesen Inhalten auch die Tonartsfolge? Nr. 1 in g-moll (Der Atlas) ist mit Nr. 2 in b-moll (Ihr Bild) durch den gemeinsamen Ton b verbunden: Revolte erstarrt $\mathrm{zu}$ »dunkeln Träumen«. Nr. 3 in As-dur (Das Fischermädchen) ist vom vorangehenden Lied im Sinne illusionär glückhafter Erinnerung vom vorherigen Lied abgetrennt, wird aber, durch zwei gemeinsame Töne (c und es), eng mit dem folgenden c-moll Stück Die Stadt verbunden. Am Meer fügt sich mit der Variante C-dur eng an. ${ }^{14}$ Damit bilden die drei Meereslieder (Nrn. 3-5) tonartlich eine Gruppe, von der sich das h-moll des Doppelgängers, dem Textinhalt entsprechend, scharf abhebt. Es ist sicherlich kein Zufall, dass diese Tonart ursprünglich auch für das letzte Lied der Winterreise vorgesehen war, aus den oben dargelegten Gründen dann aber zu a-moll abgeändert wurde.

Jedem der drei Zyklen liegt eine eigene Tonartenfolge zugrunde. Einzelne Tonarten, wie etwa A-dur in der Winterreise (Nr. 11, 19, 23) oder h-moll in der Schönen Müllerin (Nr. 16), in der Winterreise (Nr. 12 und ursprünglich auch $\mathrm{Nr}$. 24) sowie in den Heine-Liedern (Nr. 6), sind zudem als eine Art von Chiffern für bestimmte Inhalte zu verstehen, die aber erst, und dies ist wesentlich, im Kontext eines Zyklus ihre besondere Bedeutung gewinnen. Während die MüllerinnenLieder, entsprechend dem nur in diesem Zyklus einigermassen zusammenhängend fortschreitenden »Handlungsablauf« der Gedichtfolge, auch tonartlich einen Anfang (B-dur) und einen diesem diametral entgegengesetzten Schluss (E-dur) bringen, sind die andern beiden Zyklen als eine Folge gewissermassen unlogisch geordneter und nur teilweise zu Gruppen zusammengeschlossener Lieder zu verstehen. Doch gerade in dieser scheinbaren Unlogik offenbart sich Schuberts kompositorisches Denken als ein in seiner. Weise durchaus logisches, das auch mittels tonartlich parataktischer Gliederung den Bildern der Winterreise und den Heine-Texten eine zwingende zyklische Gestalt verleiht.

\section{POVZETEK}

Predmet preučevanja so Schubertovi pesemski ciklusi »Lepa mlinarica«, "Zimsko potovanje in Heinejeve pesmi, ki jih je prav tako treba razumeti kot ciklus iz skupine "Labodji spev«, kateri pa naslova ni dal Schubert. S pomočjo ugotavljanja, ali obstajajo tonalitetne zveze ali ne, poskuša avtor pokazati povezave $z$ določenimi besedili in cikličnimi grupacijami, pri čemer so $\mathrm{v}$ "Zimskem potovanju« vključene $\mathrm{v}$ preučevanje tudi transpozicije pesmi št. 6, 10, 12, 22 in 24, ki jih je že napravil Schubert. Tako se izkaže, da je osnova vsakega od treh ciklusov posebno zaporedje tonalitet. Posamezne tonalitete kot npr. A-dur v »Zimskem potovanju« (št. 11, 19, 23) ali h-mol v

14 Überdies übernimmt der erste Vorhaltsakkord von Am Meer drei Töne aus den bis zuletzt erklingenden verminderten Septakkorden von Die Stadt. 
"Lepi mlinarici« (št. 16), v »Zimskem potovanju« (št. 12 in prvotno tudi 24) in Heinejevih pesmih (št. 6) je treba razumeti kot nekakšne oznake za določene vsebine, ki pa dobijo, in to je bistveno, svoj poseben pomen šele $\mathrm{v}$ kontekstu nekega ciklusa. Medtem ko pesmi »Lepe mlinarice« kažejo ustrezno le $\mathrm{v}$ tem ciklusu razmeroma smiselno potekajočemu dejanju - tudi tonalitetno začetek (B-dur) in temu diametralen konec (E-dur), je treba oba druga cikla razumeti kot zaporedje $v$ nekem smislu nelogično urejenih in le deloma $\mathrm{v}$ skupine strnjenih pesmi. Vendar prav $\mathrm{v}$ tej navidezni nelogičnosti se razodeva Schubertova kompozicijska miselnost kot svojstveno logična, kot nekaj, kar daje tudi s pomočjo tonalitetno parataktičnega členjenja podobam "Zimskega potovanja» in Heinejevim pesmim prepričljivo ciklično. obliko. 